\title{
RENORMALIZABLE LAGRANGIANS FOR MASSIVE YANG-MILLS FIELDS
}

\author{
G.'t HOOFT \\ Institute for Theoretical Physics, University of Utrecht
}

Received 13 July 1971

\begin{abstract}
Renormalizable models are constructed in which local gauge invariance is broken spontaneously. Feynman rules and Ward identities can be found by means of a path integral method, and they can be checked by algebra. In one of these models, which is studied in more detail, local SU(2) is broken in such a way that local U(1) remains as a symmetry. A renormalizable and unitary theory results, with photons, charged massive vector particles, and additional neutral scalar particles. It has three independent parameters.

Another model has local $\mathrm{SU}(2) \otimes \mathrm{U}(1)$ as a symmetry and may serve as a renormalizable theory for $\rho$-mesons and photons.

In such models electromagnetic mass-differences are finite and can be calculated in perturbation theory.
\end{abstract}

\section{INTRODUCTION}

In a preceding article [1], henceforth referred to as I, it has been shown that, owing to their large symmetry, mass-less Yang-Mills fields may be renormalized, provided that a certain set of Ward identities is not violated by renormalization effects. With this we mean that anomalies like those of the axial current Ward identities in nucleon-nucleon interactions [2-4], which are due to an unallowed shift of integration variables in the "formal" proof, must not occur. In I it is proved that such anomalies are absent in diagrams with one closed loop, if there are no paritychanging transformations in the local gauge group. We do know an extension of this proof for diagrams with an arbitrary number of closed loops, but it is rather involved and we shall not present it here.

Thus, our prescription for the renormalization procedure is consistent, so the ultraviolet problem for mass-less Yang-Mills fields has been solved. A much more complicated problem is formed by the infrared divergencies of the system. Weinberg [5] has pointed out that, contrary to the quantum electrodynamical case, this problem cannot merely be solved by some closer contemplation of the measuring process. The disaster is such that the perturbation expansion breaks down in the infrared region, so we have no rigorous field theory to describe what happens. 
However, although the Lagrangian is invariant under local gauge transformations, the physical solutions we are interested in may provide us with a certain preference gauge, in which these solutions take a simple form. If this is the case, then the local gauge invariance is hidden, and it is very well possible that all Yang-Mills bosons become massive vector particles [6]. We do not know whether such a thing can happen with mass-less Yang-Mills fields alone, but it surely can happen in other models, of which we present some.

In all these models additional scalar fields are introduced, which are representations of the local gauge group. If, in some gauge, these fields have a non-zero vacuum expectation value, then they may fix the gauge, either completely, or partly. In the latter case, invariance under transformations of a local subgroup of the original invariance group remains evident, and some of the Yang-Mills bosons remain mass-less.

The transition from a "symmetric" to a "non-symmetric" representation is done in a way analogous to the treatment of the $\sigma$-model by Lee and Gervais $[7,8]$. The difference is of course that we have a local invariance, and we have no symmetry breaking term in the Lagrangian.

Our result is a large set of different models with massive, charged or neutral, spin one bosons, photons, and massive scalar particles. Due to the local symmetry our models are renormalizable, causal, and unitary. They all contain a small number of independent physical parameters.

A nice feature is that in certain models the electromagnetic mass-differences are finite and can be expressed in terms of the other parameters.

In sect. 2 we give a short review of the results in the preceding paper (I) on massless Yang-Mills fields. A general procedure appears to exist for deriving Feynman rules for models with a local gauge invariance. One statement must be made on our use of path integrals here: we only apply path integral techniques in order to get some idea of what the Feynman rules and Ward-identities might be. Consistency and unitarity of the renormalized expressions must always be checked later on. This has been done for the models described in this paper.

In sect. 3 we consider $S U(2)$ gauge fields and an additional scalar isospin one boson. We show how the vacuum expectation value of this boson field can become non-zero due to dynamical effects, and how two of the Yang-Mills bosons become massive, oppositely charged, vector particles, while the third becomes an ordinary photon. Of the original scalar fields one component survives in the form of a neutral spinless particle. Interaction and gauge are formulated in such a way that the theory remains renormalizable. In sect. 4 a renormalization scheme is presented, but for a more elaborate description of the renormalization procedures we refer to I. In sect. 5 we prove that the model of sect. 3 is unitary and it is easily seen that the proof applies also to the other models.

In sect. 6 we describe an example where local invariance seems broken, while global invariance remains evident. All Yang-Mills particles get equal mass, and the model resembles very much the massive Yang-Mills field studied by other authors 
$[9,10]$ except for the presence of one extra neutral scalar boson with arbitrary mass. The model can be used to describe $\rho$-mesons as elementary particles.

In sect. 7 it is shown that our " $\rho$-meson model" can be enriched with electromagnetic interactions without destroying renormalizability or unitarity. $\rho^{\circ}-\gamma$ mixture leads to phenomena like vector-dominance.

In the appendix we formulate the Feynman rules for the various models.

\section{RESUME MASSLESS YANG-MILLS FIELDS}

The massless Yang-Mills field has been discussed in I. The Lagrangian is

$$
\mathcal{L}_{\mathrm{YM}}=-\frac{1}{4} G_{\mu \nu}^{a} G_{\mu \nu}^{a}+\mathcal{L}^{c}\left(\partial_{\mu} W_{\mu}^{a}\right)
$$

with

$$
G_{\mu \nu}^{a} \equiv \partial_{\mu} W_{\nu}^{a}-\partial_{\nu} w_{\mu}^{a}+g f_{a b c} W_{\mu}^{b} W_{\nu}^{c},
$$

where $W_{\mu}^{a}$ are the Yang-Mills field components and $f_{a b c}$ are the structure constants of the underlying gauge group. $g$ is a coupling constant.

$\mathcal{L}^{c}$ is an extra term, only depending on the divergence of the field $W_{\mu}^{a}$, and it may be chosen in an arbitrary way, thus fixing the gauge.

From (2.1) the Feynman rules may be constructed by ordinary Feynman path integral methods: the procedure is clarified in the appendix. But, because of the gauge non-invariance of $\mathcal{L}^{c}$, an extra complex ghost particle field $\varphi^{a}$ must be introduced, described by the Lagrangian

$$
\mathcal{L}_{\varphi}=-\partial_{\mu} \varphi^{* a}\left(D_{\mu} \varphi\right)^{a}
$$

where $D_{\mu}$ is the covariant derivative, defined as

$$
\left(D_{\mu} X\right)^{a} \equiv \partial_{\mu} X^{a}+g f_{a b c} W_{\mu}^{b} X^{c}
$$

Furthermore, an extra factor -1 must be inserted in the amplitude for each closed loop of $\varphi$ 's.

The $\varphi$ particles (and antiparticles) do not occur in the intermediate states in the unitarity condition, because they cancel the helicity- 0 states in the $W$-field.

The Lagrangian (2.3) is related to the behaviour under local gauge transformations of the gauge non-invariant part $\mathcal{L}^{c}$ of the Lagrangian (2.1):

$$
\mathcal{L}^{c}=\mathcal{L}^{c}\left(\partial_{\mu} W_{\mu}^{a}\right)
$$

Under an infinitesimal gauge transformation generated by $\Lambda^{a}(x)$, the quantity $\partial_{\mu} w_{\mu}^{a}$ 
transforms as:

$$
\partial_{\mu} W_{\mu}^{a^{\prime}}=\partial_{\mu} W_{\mu}^{a}-g^{-1} \partial_{\mu}\left(D_{\mu} \Lambda\right)^{a}
$$

Because the fields $W_{\mu}^{a}$ occur explicitly in the covariant derivative $D_{\mu}$ in eq. (2.5), a non-trivial Jacobian factor is needed in the gauge dependent expressions for the amplitude, which is precisely the $\varphi$-particle contribution. In appendix $A$ of $I$ it is shown how to derive the Lagrangian (2.3) from eq. (2.5).

The choice

$$
e^{c}=-\alpha\left(\partial_{\mu} W_{\mu}^{a}-C^{a}\right)^{2} ; \quad \alpha \rightarrow \infty
$$

leads to the Landau gauge for $C=0$, and from the fact that the amplitudes must be independent of $C^{a}(x)$, one can derive Ward identities. The most appropriate choice however is

$$
\mathcal{L}^{c}=-\frac{1}{2}\left(\partial_{\mu} W_{\mu}^{a}(x)-J^{a}(x)\right)^{2} .
$$

For $J=0$ we have the Feynman gauge, in which the propagators are:

$$
\frac{\delta_{\mu \nu}}{k^{2}-i \epsilon}
$$

and again one can derive Ward identities.

These Ward identities supply a unique prescription for the subtraction constants in a renormalization procedure, and from them unitarity of the system follows.

If we introduce other fields which are representations of the gauge group, then all derivatives in their Lagrangian parts must be replaced by covariant derivatives, thus ensuring local gauge invariance and unitarity.

\section{SELECTION OF A PREFERENCE GAUGE BY INTRODUCING AN ISOSPIN 1 SCALAR FIELD}

Consider the case that the local gauge group is SU(2), and suppose that an isospin one scalar field or current $X^{a}(x)$ exists which has (in a certain gauge) a non-zero vacuum expectation value [6]. Then this isovector is apt to select a preference gauge, which may be taken to be the gauge in which

$$
X^{1}(x)=X^{2}(x)=0
$$

for all $x$. However, a gauge transformation of the original system to this " $X$-field 
gauge" would in general involve a non-polynomial Jacobian factor (cf. I), thus destroying renormalizability of our model. So, in general we shall abandon the gauge (3.1), but its formal possibility indicates clearly that the components $X^{1}$ and $X^{2}$ are unobservable, and $X^{3}$ acts as a "schizon": our symmetry seems to be broken.

In our renormalizable model, $X$ is simply a boson field, and we fix the gauge by adding some functional $\mathcal{L}^{c}$ to the Lagrangian as in sect. 2 . In the symmetric representation the Lagrangian is

$$
\mathcal{L}=\mathcal{L}_{\mathrm{YM}}-\frac{1}{2}\left(D_{\mu} X\right)^{2}-\frac{1}{2} \mu^{2} X^{2}-\frac{1}{8} \lambda\left(X^{2}\right)^{2}
$$

This Lagrangian corresponds to a renormalizable theory. The last term is necessary for fixing the counterterm in divergent graphs with four $X$-lines. Thus we have three independent parameters $g, \mu$ and $\lambda$.

In order to get some insight in what might happen let us consider the treeapproximation, that is, we disregard all graphs with closed loops. In this approximation all fields may be considered as being classical, and the vacuum corresponds to the equilibrium state, where all fields are constant and the total energy has a minimum. (If we specify the gauge, then this energy can be written as an integral over space pf an Hamiltonian density $\mathscr{x}(x)$.) In order for this vacuum to exist, the parameter $\lambda$ must be positive, but $\mu^{2}$ may have a negative value. In the latter case we expect that the $X$-field is non-zero in the equilibrium state: for slowly varying $X^{a}$, and $w_{\mu}^{a} \simeq 0$, we have

$$
\mathscr{X} \simeq \int_{\mathrm{V}} \mathrm{d} x\left(\frac{1}{2} \mu^{2} X^{2}+\frac{1}{8} \lambda\left(X^{2}\right)^{2}\right)
$$

This has a minimum for

$$
X^{a}(x)=e^{a} \sqrt{\frac{-2 \mu^{2}}{\lambda}},
$$

with $e^{a}$ an arbitrary vector with modulus unity. By a global gauge transformation this vector $e^{a}$ can always be pointed in the $z$-direction.

If we do quantum mechanics things do not change drastically. Eq. (3.3) must be replaced by

$$
\begin{aligned}
& \left\langle 0\left|X^{a}(x)\right| 0\right\rangle=F\left(\begin{array}{l}
0 \\
0 \\
1
\end{array}\right), \\
& F=\sqrt{\frac{-2 \mu^{2}}{\lambda}}+O(g),
\end{aligned}
$$

(the parameter $\lambda$ is of order $g^{2}$ ). 
We now proceed as Lee did for the $\sigma$-model [7]. We write

$$
X^{a}(x) \equiv F\left(\begin{array}{l}
0 \\
0 \\
1
\end{array}\right)+A_{a}(x)
$$

with

$$
\left\langle 0\left|A_{a}(x)\right| 0\right\rangle \equiv 0
$$

The Lagrangian (3.2) then becomes

$$
\begin{aligned}
\mathcal{L}= & \mathcal{L}_{\mathrm{YM}}-\frac{1}{2}\left(D_{\mu} A\right)^{2}-\frac{1}{2} \lambda F^{2} A_{3}^{2}-\frac{1}{2} g^{2} F^{2}\left(W_{\mu}^{12}+W_{\mu}^{22}\right)-\frac{1}{2} \lambda F A^{2} A_{3}-\frac{1}{8} \lambda\left(A^{2}\right)^{2} \\
& -g^{2} \dot{F} A_{3}\left(W_{\mu}^{12}+W_{\mu}^{2}\right)+g^{2} F W_{\mu}^{3}\left(A_{1} W_{\mu}^{1}+A_{2} W_{\mu}^{2}\right)-\beta\left(\frac{1}{2} A^{2}+F A_{3}\right) \\
& +g F\left(A_{1} \partial_{\mu} W_{\mu}^{2}-A_{2} \partial_{\mu} W_{\mu}^{1}\right),
\end{aligned}
$$

where

$$
\beta \equiv \mu^{2}+\frac{1}{2} \lambda F^{2}
$$

Note, that the "tadpole condition" (3.6) implies that $\beta=0$ in first order of $g$ and $\lambda$, in accordance with eq. (3.4).

We deliberately have not yet specified the local gauge. We have seen that the gauge (3.1) is no good, because it renders the theory unrenormalizable. One could very well proceed like in sect. 2 and choose the local gauge by adding

$$
\mathcal{L}^{c}=\mathcal{L}^{c}\left(\partial_{\mu} W_{\mu}\right)=-\frac{1}{2}\left(\partial_{\mu} W_{\mu}^{a}-J_{a}(x)\right)^{2},
$$

but the resulting Feynman rules are rather complicated and a massless ghost remains. It is more convenient to choose:

$$
\begin{aligned}
\mathcal{L}^{c}= & -\frac{1}{2}\left(\partial_{\mu} W_{\mu}^{3}-J_{3}(x)\right)^{2}-\frac{1}{2}\left(\partial_{\mu} W_{\mu}^{1}-g F A_{2}-J_{1}(x)\right)^{2} \\
& -\frac{1}{2}\left(\partial_{\mu} W_{\mu}^{2}+g F A_{1}-J_{2}(x)\right)^{2} .
\end{aligned}
$$

In here the functions $J_{a}(x)$ will be put equal to zero, but the fact that all physical amplitudes are independent of them enables us to formulate Ward-identities.

The fields in eq. (3.8) transform as follows under infinitesimal local gauge trans- 
formations:

$$
\begin{gathered}
\partial_{\mu} W_{\mu}^{3^{\prime}}=\partial_{\mu} W_{\mu}^{3}-g^{-1} \partial_{\mu}\left(D_{\mu} \Lambda\right)^{3} ; \\
\left(\partial_{\mu} W_{\mu}^{1}-g F A_{2}\right)^{\prime}=\partial_{\mu} W_{\mu}^{1}-g F A_{2}-g^{-1} \partial_{\mu}\left(D_{\mu} \Lambda\right)^{1}+g F^{2} \Lambda^{1}-g F \epsilon_{2 b c} \Lambda^{b} A_{c} \\
\left(\partial_{\mu} W_{\mu}^{2}+g F A_{1}\right)^{\prime}=\partial_{\mu} W_{\mu}^{2}+g F A_{1}-g^{-1} \partial_{\mu}\left(D_{\mu} \Lambda\right)^{2}+g F^{2} \Lambda^{2}+g F \epsilon_{1 b c} \Lambda^{b} A_{c} .
\end{gathered}
$$

With the same procedure as in sect. 2 we derive the $\varphi$-ghost Lagrangian $\ddagger$ :

$$
\begin{aligned}
\mathcal{L}_{\varphi}= & -\partial_{\mu} \varphi^{* a}\left(D_{\mu} \varphi\right)^{a}-g^{2} F^{2}\left(\varphi^{* 1} \varphi^{1}+\varphi^{* 2} \varphi^{2}\right) \\
& +g^{2} F\left(\varphi^{* 1} \epsilon_{2 b c} \varphi^{b} A_{c}-\varphi^{* 2} \epsilon_{1 b c} \varphi^{b} A_{c}\right)
\end{aligned}
$$

Because of our choice (3.8) for $\mathcal{L}^{c}$ the last term in (3.7) is cancelled; likewise the term

$$
+\frac{1}{2}\left(\partial_{\mu} W_{\mu}^{a}\right)^{2} \quad \text { in } \quad-\frac{1}{4} G_{\mu \nu}^{a} G_{\mu \nu}^{a} .
$$

Let us finally replace the three parameters by

$$
\begin{aligned}
& M \equiv g F>0, \\
& \alpha \equiv \lambda / g^{2}>0,
\end{aligned}
$$

and $g$.

The resulting theory has two massive, charged vector particles $W_{\mu}^{1} \pm i W_{\mu}^{2}$, with the propagator

$$
\frac{\delta_{\mu \nu}}{k^{2}+M^{2}-i \epsilon}
$$

a massless photon $W_{\mu}^{3}$, with the propagator

$$
\frac{\delta_{\mu \nu}}{k^{2}-i \epsilon}
$$

and a neutral, scalar particle $A_{3}$, with mass $M \sqrt{\alpha}$.

There are two different types of ghosts:

¥ Note that this expression is not Hermitian; the $\varphi$-ghost restores unitarity. Because of these features Feynman rules must be derived by path integral methods. The heuristic Feynman rules are here the correct ones, as is shown in the appendix. 
First: the complex $\varphi$-ghost with the oriented propagators: $\left(k^{2}+M^{2}-i \epsilon\right)^{-1}$ for $\varphi^{1,2}$ and $\left(k^{2}-i \epsilon\right)^{-1}$ for $\varphi^{3}$.

A minus sign must be inserted for each closed loop of $\varphi$-ghosts.

Second: the real $A^{1,2}$ ghosts, with no minus sign, and mass $M^{2}$ (resulting from a contribution of $\mathcal{L}^{c}$, eq. (3.8)).

These, and all other Feynman rules, except for the above mentioned minus sign, may be derived with ordinary Feynman path-integral techniques, from the Lagrangian

$$
\begin{aligned}
& \mathcal{L}=-\frac{1}{4} G_{\mu \nu}^{a} G_{\mu \nu}^{a}-\frac{1}{2}\left(\partial_{\mu} W_{\mu}^{a}\right)^{2}+\mathcal{L}_{\varphi}-\frac{1}{2}\left(D_{\mu} A\right)^{2}-\frac{1}{2} M^{2}\left(W_{\mu}^{l^{2}}+W_{\mu}^{2^{2}}\right) \\
& -\frac{\alpha M^{2}}{2} A_{3}^{2}-\frac{M^{2}}{2}\left(A_{I}^{2}+A_{2}^{2}\right)-\frac{\alpha}{2} g M A_{a}^{2} A_{3}-\frac{\alpha g^{2}}{8}\left(A_{a}^{2}\right)^{2}-g M A_{3}\left(W_{\mu}^{l^{2}}+W_{\mu}^{2^{2}}\right) \\
& +g M W_{\mu}^{3}\left(A_{1} W_{\mu}^{1}+A_{2} W_{\mu}^{2}\right)-\beta\left(\frac{1}{2} A^{2}+\frac{M}{g} A_{3}\right)+J_{3}(x) \partial_{\mu} W_{\mu}^{3}+J_{l}(x) \\
& \times\left[\partial_{\mu} W_{\mu}^{1}-M A_{2}\right]+J_{2}(x)\left[\partial_{\mu} W_{\mu}^{2}+M A_{1}\right]-\frac{1}{2} J_{a}^{2}(x)
\end{aligned}
$$

with

$\mathcal{L}_{\varphi}=-\partial_{\mu} \varphi^{* a}\left(D_{\mu} \varphi\right)^{a}-M^{2}\left(\left|\varphi^{1}\right|^{2}+\left|\varphi^{2}\right|^{2}\right)+g M\left(\varphi^{* 1} \epsilon_{2 b c}-\varphi^{* 2} \epsilon_{1 b c}\right) \varphi^{b} A_{c}$.

The functions $J_{a}(x)$ are arbitrary, which enables us to formulate Ward identities. They may be chosen to be zero. The constant $\beta$ must be adjusted in such a way, that the tadpole condition (3.6) holds.

The complex $\varphi$-particles with their unphysical "Fermi statistics", and the $A^{ \pm}$particles, with positive definite metric, must all be considered as ghosts. The most compelling reason for this is the unitarity condition; in sect. 5 we derive that these particles cancel the unphysical polarisation directions of the $W$-fields in the intermediate states, resulting from the anomalous propagators (3.12) and (3.13).

\section{RENORMALIZATION}

The expression (3.8) for $\mathcal{L}^{c}$, has especially been chosen in order to acquire the simple, quadratically convergent propagators (3.12) and (3.13), and to arrive at Feynman rules which are those of a renormalizable theory.

However, renormalization requires the introduction of a cut-off procedure, and in general this modifies the theory such that the symmetry, and therefore also unitarity, get lost. Thus, the cut-off procedure must be chosen in accordance with our symmetry requirement. To this purpose we can use the observation that in all orders of $g$ the physical amplitudes must be independent of the source function $J_{a}(x)$. The Feynman rules for the contribution of their Fourier transform $J_{d}(k)$ are given in 


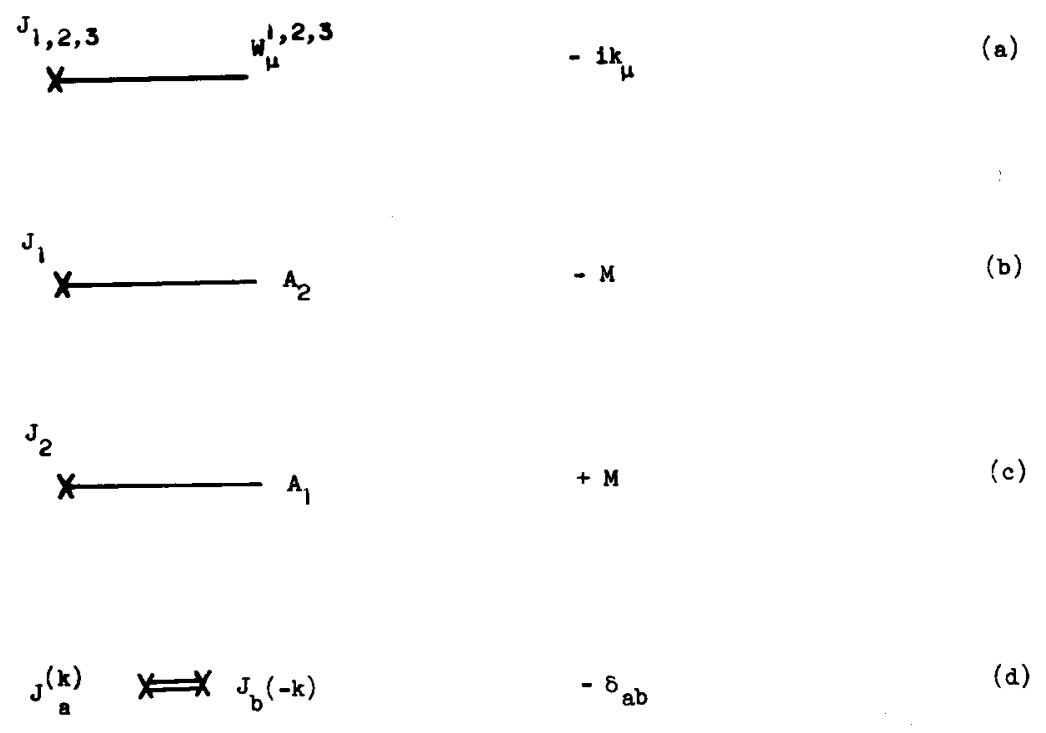

Fig. 1. Feynman rules for the contribution of the source function $J_{a}$ to the amplitude.

fig. 1 (compare eq. (3.14)). (Note that (d) is cancelled by contributions of bare $W$ and $A$ particles.)

A graphical notation for the Ward identities is shown in fig. 2. The number of " $J$-lines" must be non-zero. A combinatorial proof of these Ward identities can be given in the same way as in the case of the massless Yang-Mills fields.

Further, also in this model, a variation upon these identities can be found for the case that one of the external $W$-lines on mass shell has a non-physical polarization direction (fig. 3). The identity in fig. 3 can be proven either by combinatorics, or by using a formulation in terms of path integrals: one must consider an infinitesi-

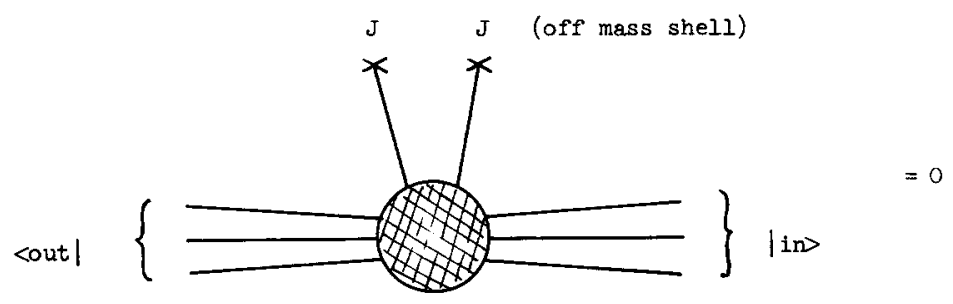

Fig. 2. Example of Ward identity expressing the fact that physical amplitudes are independent of the $J$-source. 


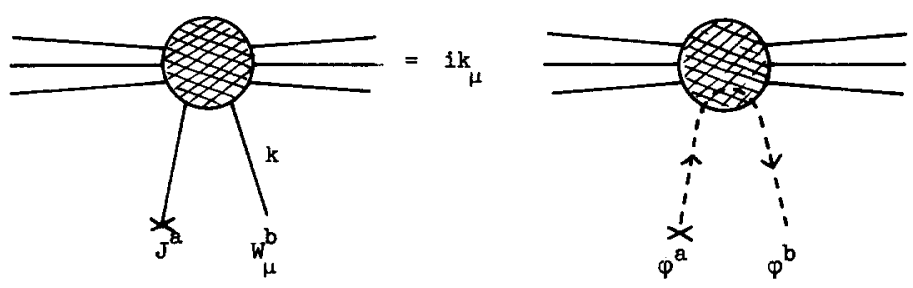

Fig. 3. Ward identity for the case that one of the external $W$-lines on mass shell has a nonphysical polarization direction.

mal gauge transformation generated by $\Lambda^{a}(x)$ with the property

$$
\partial_{\mu} D_{\mu} \Lambda(x)=J(x)
$$

$(J(x)$ being also infinitesimal).

One of the simplest ways to use these Ward identities to calculate the renormalized higher order corrections to the amplitudes, is to apply subtracted dispersion relations. The behaviour of the amplitudes for the momenta going to infinity is prescribed by the condition that renormalizability must not be destroyed at higher orders: hence, the amplitude for a diagram with $\mathrm{N}$ outgoing boson lines must behave at infinity like $(k)^{4-N} L(k)$, where $(k)$ stands for the momenta of the outgoing lines, and $L$ is a logarithmic factor. Thus the number of subtractions has been determined, whereas the Ward identities give a large restriction on the possible values of the subtraction constants. The procedure sketched here can be proven to be consistent as soon as some gauge covariant set of regulators is found. Such a set can indeed be formulated for diagrams with one closed loop by the introduction of a fifth dimension in Minkowsky space (cf. I). By introducing more dimensions one can give a consistency proof for all orders, but we shall not present it here.

\section{UNITARITY}

The equations shown in figs. 2 and 3 may be used to prove unitarity of the model. The procedure is analogous to the proof given in (I). The proof that the contributions of the $\varphi^{3}$ and $\bar{\varphi}^{3}$ intermediate states cancel those of the unphysical $W^{3}$-states is the same as in I and will not be repeated here. Actually, it is enough to show that the residues of the poles at $k^{2}=0$ of these propagators cancel.

As to the charged particle states, a unitary field theory of massive vector particles would have propagators

$$
\frac{\delta_{\mu \nu}+\frac{k_{\mu} k_{\nu}}{M^{2}}}{k^{2}+M^{2}-i \epsilon}
$$


instead of (3.12). Hence, the $W^{1,2}$ propagators have anomalous parts

$$
-\frac{k_{\mu} k_{\nu}}{M^{2}\left(k^{2}+M^{2}-i \epsilon\right)}
$$

Now, indeed, we see that as a consequence of the equations in figs. 2 and 3 , the residues of the poles at $k^{2}=-M^{2}$ of the unphysical propagators cancel. In fig. 4 it is shown which combination of these propagators has to be considered in order to prove this cancellation. (Note the explicitly written minus sign for the $\varphi$-loops; the integration over $k$ has not yet been carried out.) For more details of this proof we refer to the treatment of the analogous case in massless Yang-Mills fields, given in (I). It is because of this phenomenon that we can consider the anomalous part of the $W$-field, and the $A_{1}, A_{2}$ and $\varphi$-particles, as unphysical. They can be left out of the intermediate states without invalidating the unitarity equation.
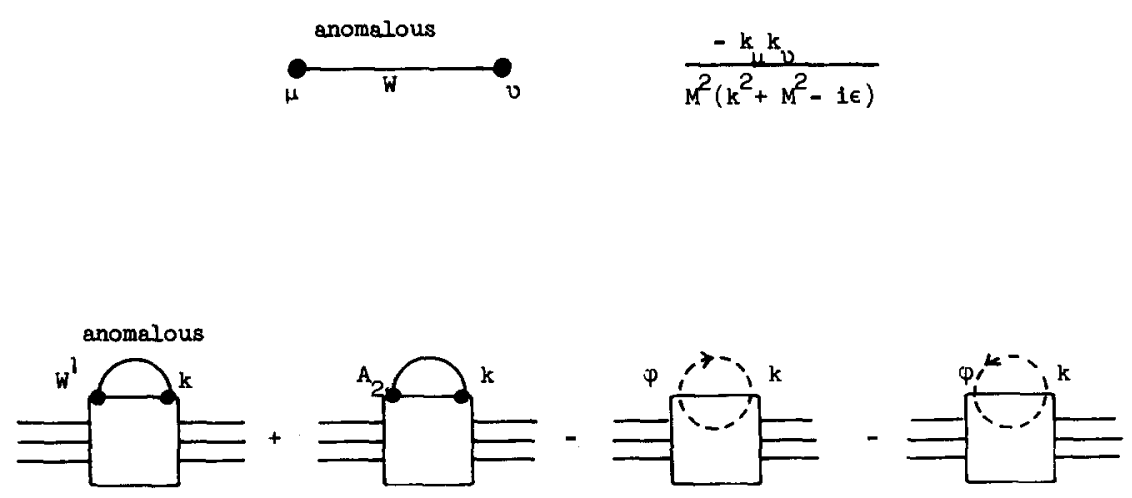

Fig. 4. Combination of unphysical propagators for fixed value of $k$.

\section{ISOSPIN- $\frac{1}{2}$ SCALAR FIELD}

Our most important conclusion from the foregoing is that a basic principle like gauge invariance can lead to renormalizable, unitary theories with massive, charged, vector particles. Many different models may be constructed this way. We like to mention in more detail a very interesting case: local SU(2)-gauge invariance with an isospin- $\frac{1}{2}$ "symmetry breaking" field

$$
K(x) \equiv\left(\begin{array}{c}
K_{1}^{\prime}+i K_{1}^{\prime \prime} \\
K_{2}^{\prime}+i K_{2}^{\prime \prime}
\end{array}\right)
$$


Let the Lagrangian in the symmetric representation be

$$
\mathcal{L}=\mathcal{L}_{\mathrm{YM}}-\left(D_{\mu} K\right)^{*} D_{\mu} K-\mu^{2} K^{*} K-\frac{1}{2} \lambda\left(K^{*} K\right)^{2}
$$

The covariant derivative of an isospin- $\frac{1}{2}$ field is:

$$
D_{\mu} K \equiv \partial_{\mu} K-\frac{1}{2} i g \tau^{a} W_{\mu}^{a} K
$$

For negative values of $\mu^{2}$, the field $K$ is expected to have a non-zero vacuum expectation value, which by a suitable global rotation in isospin space can be taken to be:

$$
\langle 0|K(x)| 0\rangle \equiv F\left(\begin{array}{l}
1 \\
0
\end{array}\right)
$$

Now, it appears to be convenient to express the complex spinor $K$ in terms of a real isospin singlet and a real triplet:

$$
\begin{aligned}
K & \equiv F\left(\begin{array}{l}
1 \\
0
\end{array}\right)+\frac{1}{\sqrt{2}}\left(Z+i \psi_{a} \tau^{a}\right)\left(\begin{array}{l}
1 \\
0
\end{array}\right), \\
& =F\left(\begin{array}{l}
1 \\
0
\end{array}\right)+\frac{1}{\sqrt{2}}\left(\begin{array}{c}
Z+i \psi_{3} \\
-\psi_{2}+i \psi_{1}
\end{array}\right),
\end{aligned}
$$

and to introduce the independent parameters

$$
\begin{aligned}
M^{2} & \equiv \frac{1}{2} g^{2} F^{2}, \\
\alpha & =\lambda / g^{2},
\end{aligned}
$$

and $g$.

In this representation, the Lagrangian is:

$$
\begin{aligned}
\mathcal{L}= & -\frac{1}{4} G_{\mu \nu}^{a} G_{\mu \nu}^{a}+\mathcal{L}^{c}-\frac{1}{2} M^{2} W_{\mu}^{2}-\frac{1}{2}\left(\partial_{\mu} Z\right)^{2}-\frac{1}{2}\left(\partial_{\mu} \psi\right) D_{\mu} \psi-\frac{1}{2} 4 \alpha M^{2} Z^{2} \\
& +\frac{1}{2} g W_{\mu}^{a}\left(Z \partial_{\mu} \psi_{a}-\psi_{a} \partial_{\mu} Z\right)-\frac{1}{8} g^{2} W_{\mu}^{2}\left(\psi^{2}+Z^{2}\right)-\frac{1}{2} g M W^{2} Z-\alpha M g Z\left(\psi^{2}+Z^{2}\right) \\
& -\frac{1}{8} \alpha g^{2}\left(\psi^{2}+Z^{2}\right)^{2}-\beta\left[\frac{1}{2}\left(Z^{2}+\psi^{2}\right)+\frac{2 M}{g} Z\right]-M \psi_{a} \partial_{\mu} W_{\mu}^{a},
\end{aligned}
$$

where the parameter $\beta \equiv \mu^{2}+\lambda F^{2}$ must be chosen in such a way that all tadpoles cancel. It is of order $g^{2}$. 
$\mathcal{L}^{c}$ is chosen to be

$$
\mathcal{L}^{c}=-\frac{1}{2}\left(\partial_{\mu} W_{\mu}^{a}-M \psi_{a}-J_{a}\right)^{2}
$$

so that again the Feynman propagator (3.12) for the $W$-field emerges, and the last term of eq. (6.6) cancels.

By studying the behaviour of $\mathcal{L c}^{c}$ under gauge transformations we derive the Lagrangian for the ghost field $\varphi$ (compare sects. 2 and 3 )

$$
\mathcal{L}_{\varphi}=-\partial_{\mu} \varphi^{*} D_{\mu} \varphi-M^{2} \varphi^{*} \varphi-\frac{1}{2} M g \varphi^{*} \varphi Z+\frac{1}{2} M g \epsilon_{a b c} \varphi^{* a} \varphi^{b} \psi^{c}
$$

The Feynman rules for the source function contributions are now those of fig. 5 .

Now we observe that the Lagrangian (6.6) as well as the Ward identities remain invariant under global isospin transformations, if the $\psi$ fields are considered as a triplet and the $Z$ as a singlet. Only local gauge invariance has been broken. Here the $\psi$ fields act as additional ghosts, and all three isospin components of the $W_{\mu}^{a}$-fields have become massive. The $Z$ is an additional physical particle.

Many authors $[9,10]$ have considered the massive Yang-Mills theory described by the Lagrangian

$$
\mathcal{L}_{\mathrm{YM}}-\frac{1}{2} M^{2} W_{\mu}^{2}
$$

That model appears to be non renormalizable, although many of the divergencies can be seen to cancel by the introduction of two kinds of ghost fields $[11,12]$. In our model also two ghosts appear: the complex $\varphi$-field, with Fermi statistics, and the $\psi$-field. But now we see that the introduction of one physical isospin-zero particle $Z$ can render the model renormalizable. Its mass is a new independent parameter. For large values of this mass we get something very similar to the old model (6.9).
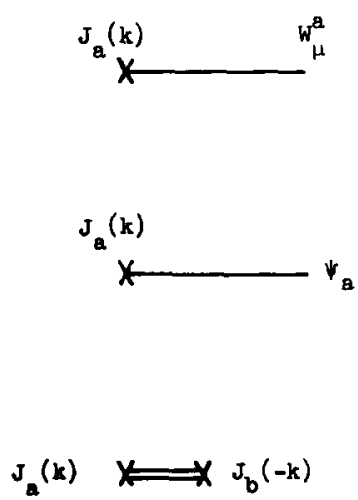

Fig. 5. Feynman rules for the $J$-source contribution to the amplitude. 


\section{ISOSPIN AND ELECTROMAGNETISM; VECTOR DOMINANCE}

In the previous model, electromagnetism can be introduced in an elegant way $\dagger$. Consider first the symmetric representation (6.1). Let us assume the presence of a "hyperelectromagnetic" field, $\widetilde{A}_{\mu}$, which does not break isospin. Let in (6.1) only the K particle have a "hypercharge" $q$. The Lagrangian is then:

$$
\mathcal{L}=\mathcal{L}_{\mathrm{YM}}-\frac{1}{4} \tilde{F}_{\mu \nu} \tilde{F}_{\mu \nu}-\left(\widetilde{D}_{\mu} K\right)^{*} \widetilde{D}_{\mu} K-\mu^{2} K^{*} K-\frac{1}{2} \lambda\left(K^{*} K\right)^{2},
$$

with

$$
\begin{gathered}
\tilde{F}_{\mu \nu} \equiv \partial_{\mu} \tilde{A}_{\nu}-\partial_{\nu} \tilde{A}_{\mu}, \\
\tilde{D}_{\mu} K \equiv D_{\mu} K+i q \tilde{A}_{\mu} K
\end{gathered}
$$

The gauge group in this model is $\mathrm{SU}(2) \otimes \mathrm{U}(1)$. Let us consider an infinitesimal gauge transformation:

$$
\begin{aligned}
K^{\prime} & =\left(1-\frac{1}{2} i \Lambda^{a} \tau^{a}-i \tilde{\Lambda}\right) K, \\
W_{\mu}^{\prime a} & =W_{\mu}^{a}-g^{-1}\left(D_{\mu} \Lambda\right)^{a}, \\
\tilde{A}_{\mu}^{\prime} & =\tilde{A}_{\mu}+q^{-1} \partial_{\mu} \tilde{\Lambda}
\end{aligned}
$$

where $\Lambda^{a}(x), \tilde{\Lambda}(x)$ are generators of an infinitesimal gauge transformation.

Now if the $K$ field has a non-zero vacuum expectation value:

$$
\langle 0|K(x)| 0\rangle=F\left(\begin{array}{l}
1 \\
0
\end{array}\right)
$$

then the physical fields will only appear to be invariant under those transformations (7.2) that leave the spinor

$$
\left(\begin{array}{l}
1 \\
0
\end{array}\right)
$$

invariant; that are the transformations with

$$
\Lambda^{1}=\Lambda^{2}=0 ; \quad \Lambda^{3}=-2 \widetilde{\Lambda} \equiv \Lambda^{\mathrm{EM}}
$$

† The model of this section is due to Weinberg [13], who showed that it can describe weak interactions between leptons. His lepton model can be shown to be renormalizable. 
Let us call such transformations electromagnetic gauge transformations. If we define

$$
\begin{aligned}
& w_{\mu}^{1,2} \equiv \rho_{\mu}^{1,2} \\
& W_{\mu}^{3} \equiv \rho_{\mu}^{3}+\frac{2 q}{g} \tilde{A_{\mu}} \\
& K \equiv F\left(\begin{array}{l}
1 \\
0
\end{array}\right)+\frac{1}{\sqrt{2}}\left(\begin{array}{l}
Z+i \psi_{3} \\
-\psi_{2}+i \psi_{1}
\end{array}\right)
\end{aligned}
$$

then these quantities transform under electromagnetic gauge transformations like:

$$
\begin{aligned}
& Z^{\prime}=Z \text {, } \\
& \psi_{3}^{\prime}=\psi_{3}, \\
& \psi_{1}^{\prime} \pm i \psi_{2}^{\prime}=\mathrm{e}^{ \pm i \Lambda} \mathrm{EM}\left(\psi_{1} \pm i \psi_{2}\right) \\
& \rho_{\mu}^{3^{\prime}}=\rho_{\mu}^{3},
\end{aligned}
$$

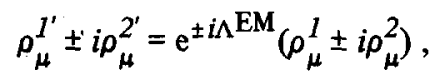

$$
\begin{aligned}
& \tilde{A}_{\mu}^{\prime}=\tilde{A}_{\mu}-\frac{1}{2 q} \partial_{\mu} \Lambda^{\mathrm{EM}} \text {. }
\end{aligned}
$$

Finally, we make the substitutions:

$$
\widetilde{A_{\mu}} \equiv A_{\mu}\left(1+\frac{4 q^{2}}{g^{2}}\right)^{-\frac{1}{2}} ; \quad e \equiv 2 q\left(1+\frac{4 q^{2}}{g^{2}}\right)^{-\frac{1}{2}}
$$

In terms of these variables the Lagrangian (7.1) becomes:

$$
\begin{aligned}
\mathcal{L}= & -\frac{1}{4} \rho_{\mu \nu}^{a} \rho_{\mu \nu}^{a}-\frac{1}{4} F_{\mu \nu} F_{\mu \nu}-\frac{e}{2 g} F_{\mu \nu} \rho_{\mu \nu}^{3}+\mathcal{L}^{c}-\frac{1}{2} M^{2} \rho_{\mu}^{2}-\frac{1}{2}\left(\partial_{\mu} Z\right)^{2}-\frac{1}{2} 4 \alpha M^{2} Z^{2} \\
& -\frac{1}{2}\left(\partial_{\mu}^{\mathrm{EM}} \psi\right) D_{\mu}^{\mathrm{EM}} \psi+\frac{1}{2} g \rho_{\mu}^{a}\left(Z \partial_{\mu}^{\mathrm{EM}} \psi_{a}-\psi_{a} \partial_{\mu} Z\right)-\frac{1}{2} g M \rho^{2} Z-\frac{1}{8} g^{2} \rho^{2}\left(\psi^{2}+Z^{2}\right) \\
& -\alpha M g Z\left(\psi^{2}+Z^{2}\right)-\frac{1}{8} \alpha g^{2}\left(\psi^{2}+Z^{2}\right)^{2}-\beta\left(\frac{1}{2}\left(Z^{2}+\psi^{2}\right)+\frac{2 M}{g} Z\right)-M \psi_{a} \partial_{\mu}^{\mathrm{EM}} \rho_{\mu}^{a},
\end{aligned}
$$


where

$$
\begin{aligned}
\partial_{\mu}^{\mathrm{EM}} \psi_{3} & \equiv \partial_{\mu} \psi_{3}, \\
\partial_{\mu}^{\mathrm{EM}}\left(\psi_{1} \pm i \psi_{2}\right) & \equiv\left(\partial_{\mu} \pm i e A_{\mu}\right)\left(\psi_{1} \pm i \psi_{2}\right), \\
\left(D_{\mu}^{\mathrm{EM}} \psi\right)_{a} & \equiv \partial_{\mu}^{\mathrm{EM}} \psi_{a}+g \epsilon_{a b c} \rho_{\mu}^{b} \psi_{c}, \\
\rho_{\mu \nu}^{a} & \equiv \partial_{\mu}^{\mathrm{EM}} \rho_{\nu}^{a}-\partial_{\nu}^{\mathrm{EM}} \rho_{\mu}^{a}+g \epsilon_{a b c} \rho_{\mu}^{b} \rho_{\nu}^{c} .
\end{aligned}
$$

Let us choose

$$
\mathcal{L}^{c}=-\frac{1}{2}\left(\partial_{\mu}^{\mathrm{EM}} \rho_{\mu}^{a}-M \psi_{a}-J_{a}\right)^{2}-\frac{1}{2}\left(\partial_{\mu} A_{\mu}-J^{\mathrm{EM}}\right)^{2},
$$

so that the last term in (7.8) is cancelled, while the photon, and the $\rho$-particle have the Feynman propagators (3.12) and (3.13) resp.

The contributions of the $\varphi$-ghosts is described by

$$
\mathcal{L}_{\varphi}=-\partial_{\mu}^{\mathrm{EM}} \varphi^{*} D_{\mu}^{\mathrm{EM}} \varphi-M^{2} \varphi^{*} \varphi-\frac{1}{2} M g \varphi^{*} \varphi Z+\frac{1}{2} M g \epsilon_{a b c} \varphi^{* a} \varphi^{b} \psi^{c}
$$

again with the additional minus sign for each closed loop of $\varphi$ 's.

So, we have arrived at a renormalizable model containing photons, $\rho$-mesons and neutral $Z$-particles. There are four independent parameters: $g, M, \alpha$ and $e$. The parameter $\beta$ is dictated by the tadpole condition:

$$
\langle 0|Z(x)| 0\rangle=0
$$

Note the third term in (7.8), which leads to phenomena like vector dominance. It is a consequence of the translation (7.5b).

\section{CALCULATION OF ELECTROMAGNETIC MASS DIFFERENCES}

One of the main virtues of the models presented here is, that there are no ambiguities due to infinities, and the number of independent parameters is small. It is interesting to calculate some "electromagnetic" mass differences. For instance, in the model of sect. 3 one may introduce an isospin one fermion $\dagger$

$$
\mathcal{L}_{N}=-\bar{N}\left(m+\gamma_{\mu} D_{\mu}\right) N
$$

$\dagger$ It appears that counterterms of the form $\bar{N}^{a} \epsilon_{a b c} X^{b} N^{c}$, or, in the asymmetric representation, $\bar{N}^{a} e_{a b c} A^{b}{ }^{b}$, are not needed for renormalization. 
A direct computation of the difference in mass of the $N^{ \pm}$and $N^{\circ}$ seems to lead to ambiguous results because the integrals for the self-energy corrections diverge. But, one of the Ward identities states that the graph of fig. 6a equals zero, if the charged particle $N_{2}$, and the neutral $N_{3}$ are both on mass shell. Let us consider all terms of this graph up to third order in $g$ (fig. 6b).

The first term only contributes if $m^{ \pm} \neq m^{\circ}$. Now the mass difference $m^{ \pm}-m^{\circ}$ will be of order $g^{2}$, so $q$ can be taken of order $g^{2}$. Hence the last four diagrams of fig. $6 b$ will not contribute in the third order of $g$.

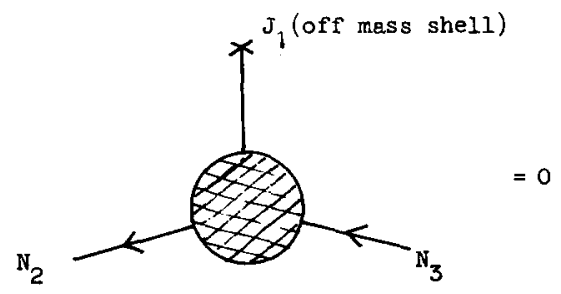

(a)
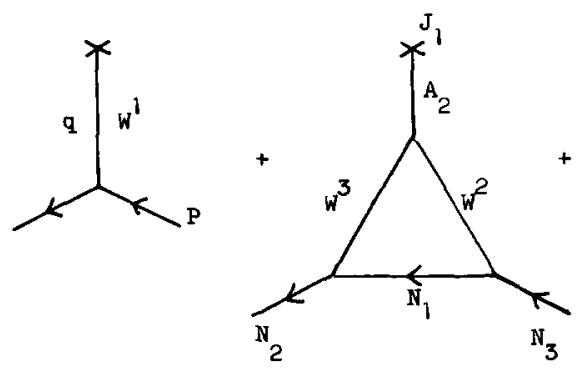

(b)
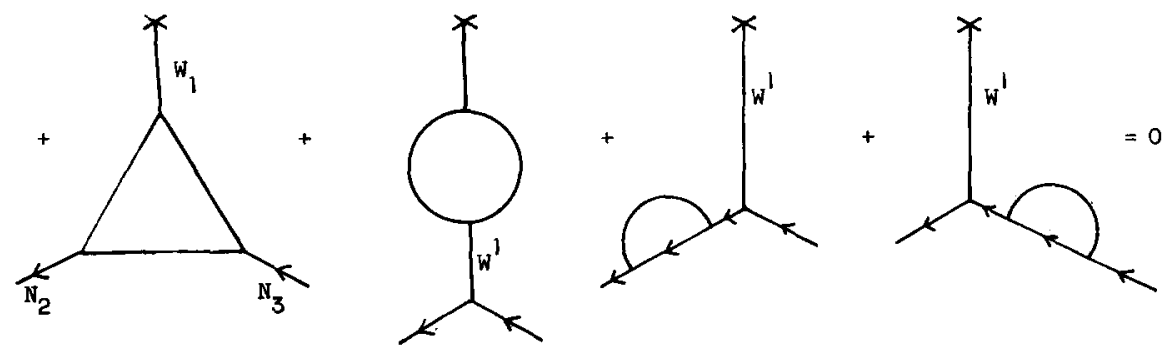

Fig. 6. a. Ward identity for the model of sect. 3, augmented with isospin-one fermions. b. All contributions up to order $g^{3}$. 
Thus, the Ward identity reads

$$
g\left(m_{ \pm}-m_{\mathrm{o}}\right)-M \cdot B=0
$$

where $B$ is the second graph of fig. $6 \mathrm{~b}$.

The momentum transfer $q$ may now be taken to be zero, and both external $N$ lines have momentum $p$ with $p^{2}=-m^{2}$. The integral in $B$ converges, and the result is:

$$
\frac{m^{ \pm}-m^{\circ}}{m}=\frac{2 g^{2}}{(4 \pi)^{2}} \int_{0}^{1} \mathrm{~d} x(1+x) \log \left[1+\frac{M^{2}(1-x)}{m^{2} x^{2}}\right]
$$

(in second order of $g$ ).

This mass-difference is always positive, and for small resp. large values of $M^{2} / m^{2}$ eq. (8.3) may be simplified to

$$
\begin{aligned}
& m^{ \pm}-m^{\circ}=\frac{g^{2} M}{8 \pi} \quad\left(M^{2} \ll m^{2}\right) ; \\
& m^{ \pm}-m^{\circ}=\frac{3 g^{2} m}{(4 \pi)^{2}} \log \frac{M^{2}}{m^{2}} \quad\left(M^{2} \gg m^{2}\right) .
\end{aligned}
$$

A negative mass-difference is found for the $\rho$-meson itself in the model of sect. 7 . This mass-difference is of zeroth order, and originates from the "vector dominance" term in eq. (7.8). Diagonalisation of the bilinear terms in (7.8) leads to the mass formula for the $\rho$-mesons (in zeroth order):

$$
M_{\mathrm{o}}^{2}=\frac{M^{ \pm}}{1-e^{2} / g^{2}}
$$

The author wishes to thank Prof. M.Veltman for his invaluable criticism and encouragement.

\section{APPENDIX}

\section{Feynman rules for the various models}

In the preceding article I a system with a complete local gauge invariance was quantized using a path integral technique. It was shown how to make the integrand gauge non-invariant without changing the total amplitude. The same procedure has been applied here. The term which breakes the gauge invariance is always called $\mathcal{L}^{c}$. As a consequence of this procedure, the Feynman rules must always be derived 
from the final Lagrangians by performing the path integral, and not by canonical quantization. This means that the propagators are always the inverse of the matrices in the bilinear terms of the Lagrangian, and the vertices are the coefficients in front of the remaining terms in the Lagrangian, regardless whether time derivatives occur or not. We mention here some of these Feynman rules.

Model of section 3. (compare (3.14) and (3.15)).

physical particles:

$$
\begin{array}{lll}
\stackrel{W^{1,2}}{\stackrel{\nu}{\mu}} & \frac{\delta_{\mu \nu}}{k^{2}+M^{2}-i \epsilon} \\
\stackrel{W^{3}}{A_{3}} & \frac{\delta_{\mu \nu}}{k^{2}-i \epsilon} & \frac{1}{k^{2}+\alpha M^{2}-i \epsilon}
\end{array}
$$

ghosts:

$$
\begin{aligned}
& A_{1,2} \ldots \frac{1}{k^{2}+M^{2}-i \epsilon} \\
& -\stackrel{\varphi}{I, 2} \rightarrow \rightarrow \frac{1}{k^{2}+M^{2}-i \epsilon} \\
& -\underline{\varphi}^{3} \rightarrow-\infty \frac{1}{k^{2}-i \epsilon}
\end{aligned}
$$

for each closed loop of $\varphi$ lines: $\quad-1$

some of the vertices:<smiles>[M]C[18O]</smiles><smiles>[M]C</smiles><smiles>[M]C(O)C(O)O</smiles>
as in massless Yang-Mills fields.

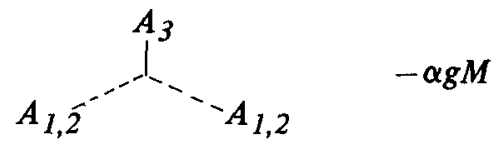


<smiles>CC(C)C</smiles>

etc.

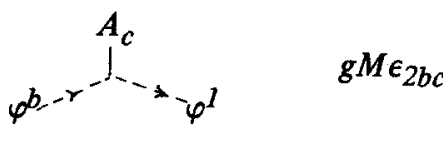<smiles>CC(C)C(=O)C(C)C</smiles>

$\underline{A_{1,2}} \ldots$

$\left.A_{3}, A_{3}\right\}-\beta$

The vertices (A.12) and (A.13) must be added to higher order tadpole- and selfenergy diagrams, with $\beta$ chosen such, that all tadpole contributions cancel.

Model of section 6. (compare (6.6), (6.7) and (6.8)).

physical particles:

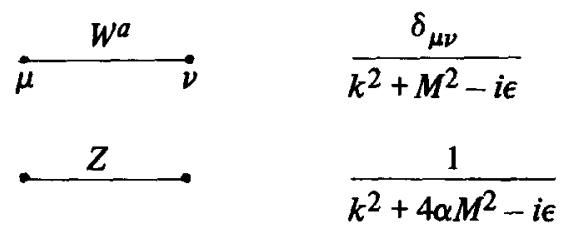

ghosts:

$$
\begin{array}{cc}
-\psi_{-}^{a} \ldots & \frac{1}{k^{2}+M^{2}-i \epsilon} \\
-\varphi_{-}^{a} & \frac{1}{k^{2}+M^{2}-i \epsilon}
\end{array}
$$


some of the vertices:

$$
\begin{aligned}
& \psi^{a-{ }_{p}}{\stackrel{1}{q} \psi^{c}}^{b} \quad-\frac{1}{2} i g \epsilon_{a b c}(p-q)_{\mu} \\
& \psi^{a-\cdots}-\psi^{b} \quad-2 \alpha M g \delta_{a b} \\
& \overbrace{Z}^{Z}-6 \alpha M g
\end{aligned}
$$

etc.

Model of section 7. (compare (7.8), (7.9), (7.10) and (7.11)).

Rules as in preceding model, but with additional photon lines:

$$
\sim \sim \sim \sim \frac{\delta_{\mu \nu}}{k^{2}-i \epsilon}
$$

and vertices:

$$
\begin{aligned}
& A_{\mu} \sim-\rho_{\mu}^{3} \quad-\frac{e}{g}\left(k^{2} \delta_{\mu \nu}-k_{\mu} k_{\nu}\right)
\end{aligned}
$$

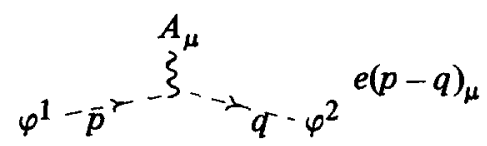

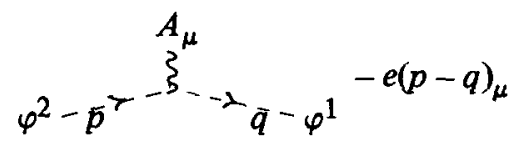

etc.

The $J$-source can now emit a photon and a $\rho$-meson simultaneously:

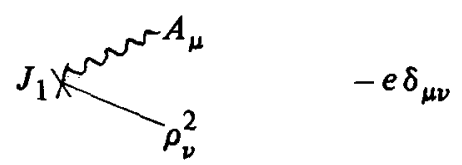

etc. 
The vertex (A.22) is a consequence of the fact that we did not diagonalize the bilinear terms in (7.8) completely. The diagonalized propagators have a rather complicated form. For small $e$ it is easier to leave (A.22) as it stands.

If necessary, vertices like (A.25) for the $J$-source contribution may be avoided by choosing another expression for $\mathcal{L}^{c}$ (eq. (7.10)).

\section{REFERENCES}

[1] G.'t Hooft, Nucl. Phys. B33 (1971) 173.

[2] J.S.Bell and R.Jackiw, Nuovo Cimento 60A (1969) 47.

[3] S.L.Adler, Phys. Rev. 177 (1969) 2426.

[4] I.S.Gerstein and R.Jackiw, Phys. Rev. 181 (1969) 1955.

[5] S.Weinberg, Phys. Rev. 140 (1965) B5 16.

[6] T.W.B.Kibble, Phys. Rev. 155 (1967) 1554.

[7] B.W.Lee, Nucl. Phys. B9 (1969) 649.

[8] J.L.Gervais and B.W.Lee, Nucl. Phys. B 12 (1969) 627.

[9] M.Veltman, Nucl. Phys. B7 (1968) 637; Nucl. Phys. B21 (1970) 288.

[10] D.G.Boulware, Ann. of Phys. 56 (1970) 140.

[11] A.A.Slavnow, L.D.Faddeyev, Massless and massive Yang-Mills field, Moscow preprint 1970.

[12] E.S.Fradkin, U.Esposito and S.Termini, Rivista del Nuovo Cimento 2 (1970) 498.

[13] S.Weinberg, Phys. Rev. Letters 19 (1967) 1264. 